\title{
The Communication Value of Multi-style Subtitles
}

\author{
Guangyu Zeng \\ Chongqing Normal University, Literature College, Chongqing, China, 401331
}

Keywords: Multi-Style Subtitles, Semiotics, Spread, Accept

\begin{abstract}
The article re-examines the communicative value of fancy subtitles in television programs in recent years from the perspective of semiotics. It is believed that fancy subtitles have the functions of activating background knowledge, marking information focus and guiding information acceptance in information dissemination. By reducing the information attenuation and disagreement of the information generated by the information asymmetry of the codification and decoding, the way of controlling the audience's selective attention and selective understanding, guiding the interpretation of the meaning of the meaning of the way, fancy subtitles to complete the television program from the audience to guide the audience to accept the change-oriented communication.
\end{abstract}

\section{Introduction}

In recent years, reality show class variety development is rapid. As one of the existential forms of television communication, the reality show of the reality show has a new comment on the use of subtitles. From the beginning of the first quarter of Hunan TV's "Daddy", there were many fancy subtitles on the screen, in addition to the language, voice language and regular subtitle language of traditional TV programs. Industry referred to as "flower word". Since 2013, fancy subtitles began to be widely used in major variety show, such as the oriental TV's "extreme challenges", Zhejiang TV "run it, brother!" and many more. The reason why this subtitle alone named "flower word", its purpose is to distinguish the general meaning of the subtitles, fancy subtitles in the television has its unique role and significance.

\section{The Acceptance Function of Fancy Subtitles}

The subtitle in the narrow sense refers only to the form of text in the sound language of the television program. From the perspective of information dissemination, the function of such subtitles is only auxiliary, and is mainly used to assist in the accurate dissemination of information. The generalized subtitles include the symbols of all the text appearing on the screen. Such as caption subtitles for annotating columns or program titles, for annotating subtitles that the audience may not understand, such as minority languages, foreign languages, and descriptive subtitles for the content of the picture and the speech that is difficult to express, for capturing captioning or informative subtitles that are not related to the program being played and subtitles of knowledge or information classes in text form as a medium. There are also scholars in accordance with the TV subtitles and television content related to the subtitles are directly related to subtitles and non-related subtitles.

The current classification of subtitles, whether from the form, function or relevance cannot be properly classified fancy subtitles. If the screenwriter of the television program is the first text processing, the sound of the program, the picture is the second text processing, then the fancy 
subtitles can count the third text processing, belonging to a sub-text, rather than belonging to the sound Language and Picture Language Auxiliary Text. It transcends the expression of the current television language (including voice language, picture language and subtitle language) in many ways.

Activate the Background Information. The reality show is different from the general TV program is that it is by a number of characters, multiple clues common in a story context. If the audience lacks relevant story clues, it is not possible to classify audiovisual information reasonably, which does not conform to our cognitive schema of understanding events. In addition, the known information of the audience is uncertain to the communicator, and if there is no proper comment, the viewer cannot receive the message to be communicated by the communicator. The other program's solution to this problem is to add annotated subtitles. For example, the film lines involved in a more remote knowledge points, then in the form of parentheses to comment on the knowledge points. This is through the text to make up for the audience may exist in the knowledge of the blind spot, and thus ensure the accuracy of information dissemination.

And fancy subtitles usually in simple text to directly activate the audience have the background information, so as to achieve the purpose of targeted information dissemination. In "run it, brother! ", Wang Zu Lan's height has been ridiculed the object, the program group often use this to render the entertainment effect. Height as Wang Zu blue uncontrollable variable factors, if used to ridicule, it is equivalent to the burden of crosstalk. Once the audience is familiar with this burden, the second re-use cannot increase the effect. Fancy subtitles do not tell the audience directly in the annotated text of "Wang Zu Lan's short", but at the appropriate time in a different text form. For example, when the program involves taking things game, the program hit the "height is Mishap" fancy subtitles, to remind the audience "Wang Zu Lan short" this information, rendering program "ridicule Wang Zu Lan height" effect.

New information into the audience after the cognitive range is no longer new information, so the same topic or program with the same annotation subtitles is difficult to trigger the audience again to generate new knowledge. Still in the "Wang Zu Lan short", for example, in many of the program, in addition to the first time, the audience to Wang $\mathrm{Zu}$ blue height for new information, the other time it has been stored as background information. At this point, the audience in the process of communication will not intentionally notice this information. Fancy subtitles can re-appear at the appropriate time and re-activate the background information stored in the brain, once again to achieve the effect of targeted information dissemination. For example, the program involved in the case of Wang $\mathrm{Zu}$ blue punishment, because others from the top of the top of the penalty props far, the results lighter than other players. At this time, fancy subtitles "born my material will be useful" to activate the audience "height is Mishap" "Wang Zu Lan short" and other background information, and then the audience through their own understanding, winding streets to receive the program at this time To spread out the information, thereby enhancing the dissemination of results.

Mark the Focus Information. The focus of information is the subject of linguistic research, and linguistic research shows that language is one of the tools to carry and disseminate information. At the language level, the focus of information is usually through the voice (such as stress, pause), non-linear components for marking and semantic components of the extraordinary coordination and other means to highlight the focus of information dissemination.

Unlike the language symbol is a linear system, the television symbol is a combination of image symbols, sound symbols and text symbols, unlike any single symbolic system. But in the information dissemination point of view, the two have in common. According to the definition of linguistic symbols by Ferdinand de Saussure, we can simplify the process of linguistic information 
dissemination: the information coder encodes the signifier as a signifier, which means that through the channel and noise, the information the receiver decodes it as referring. The ideal process is the correctness that the coders have compiled. The reality is that in this process, the information is not completely received, or the decoder is not receiving the information transmitted by the encoder.

The semiotics suggests that the information of the objective world is encoded as a symbol carrying artificial senses, so that the information undergoes a symbolic process in the process of communication. Charles Sanders Peirce, known as the father of semiotics, divides the symbol into three categories: reconstituted, object, and explanatory. In addition to the corresponding Taussure's "signifier" and "signified", the "explanatory" emphasizes that meaning is not independent, but depends on the interpretation of the main body of the symbol, so that even if the same object, different interpretation of the air subject, interpretation of the meaning will be different.

TV symbols are the fusion of multiple symbolic systems, and the information that the communicator wants to propagate is transmitted to the audience after encoding, and the subject may have different results due to subjective differences. Especially the screen information, it is not a linear way to spread, but the overall information encoded as a plane, a one-time presentation to the audience. When the audience encounters such image information, it often relies on the position of the lens focus (which is also the point of information the communicator wants to transmit) to obtain information. Of course, the ideal communication effect is so, but no one can guarantee that the audience is indeed correct decoding.

Fancy subtitles of course cannot decide how the audience decoding, but it is on the audience to what information decoding, how decoding has an important role. The way is to mark the focus of information, to guide the audience in many possible information to accurately find the location of the decoding and decoding. For example, in the "run it, brother!" "Program, running men's team played, surrounded by a lot of people onlookers, Deng Chao when the flower is" welcome to see our concert ", Chen He appeared when" I am more and more famous it. "Originally, when the players played, the audience may be concerned about their clothing, to say, ride the means of transport and so on the possible elements, and the flower mark makes the audience will focus on their appearance when the appearance of the two Different "flower word" is to guide the audience concerned about Deng Chao and Chen He in the face of many crowd when the different reactions. Another situation is that the screen information itself does not have the natural focus, need to mark the flower to make it a contrast focus. For example, Deng Chao in a certain period of time inadvertently raised a small finger, this information is not focused on the enlarged picture, it is difficult to be captured by the audience. However, the program group in his late little finger with the flower word "orchid finger", all of a sudden the attention of the audience attracted to this action, as one of the laughter of the program.

Some of the sound symbols may be ignored by the audience and the communicator would like to convey the information, but also need to mark the word. "Daddy go" in the first quarter, Tian Liang called his daughter Cindy English name with a clear dialect accent, but as a voice message, fleeting, the audience may not have this sensitive, this time flower word " "With the next to remind the audience to take the initiative to decode the information point. Similarly, Deng Chao in the men's team called "school tyrants" and then in its English "We are family." With a strong Chinese accent, by the program group later coupled with "logging tired" flower word, is also marked one of the highlights of the information. In the ordinary dialogue subtitles will need to emphasize or convey the new text of the text part of the highlight is also a mark approach.

Guide the Information Acceptance. As mentioned above, the television symbol as a complex of multiple symbolic systems, under the "interpretation of" the role of the communicator intended to 
spread the information is likely to produce variants in the receiving side, the dissemination of the effect and therefore greatly reduced. Flower is mainly used in the reality show or entertainment strong variety show, this program is characterized by information points scattered, less new information to entertainment-based. If there is no flower word, a lot of reality show class into a short life in the parents, in the audience, in addition to be able to snoop the star of ordinary life, there is no value. Therefore, the flower word in the reality shows class variety play in the guide information to accept the role. All the characters in the reality show are starring, and each person has its own unique personality, in the limited communication time, need to pass to the audience enough effective information. The floral word can be "hinted" by the symbol "what character is a character of the audience, and can also" guide "the viewer to understand the picture according to the description of the flower word. For example, Sun Honglei in the "extreme challenges" program, complete the task from the vault to take the gold bullion, but he took very little, this time on the image with the flower word "light", and the next lens Wang Xun holding full a box of heavy gold bars contrast, flower word "light" is to guide the audience to do the purpose of Sun Honglei speculation.

For some elements such as psychological activities, characters, behavioral motives, emotional attitudes and so on through the screen and the sound easily lead to personalized interpretation of the elements, the role of the flower is to determine a certain interpretation method to control the direction of the audience decoding. From the perspective of semiotics, this pattern is intended to minimize the amount of information that the communicator propagates in the encoding process to the receiver to the signifier. In other words, the communicator wants the recipient to receive only the information they want to spread. This information is even deviated from the original sound or pictorial symbols carrying information into a language independent of the voice language and language outside the third language, that is, fancy subtitles itself is the source of information. "Son-in-law" program sinking and aunt chat through the language symbol mildly conveyed aunt forced marriage information, the audience may be decoding information at this time there is an infinite number of possible. But at this time the flower word "ginger or old spicy" appears, direct the audience to comment on this dialogue. This is equivalent to determining the uncertainty of countless viewers as one.

\section{The Acceptance of Fancy Subtitles-Oriented Transmission}

Subtitles were first used in television to identify the title, and then extended to replace the commentary or voice over the text form, used to annotate the screen in the time, place, character information, and then developed with the characters synchronized language symbol form. To this step, the subtitles are only in the surface of the program feel the state, and will not interfere with the audience decoding of the program. To the program visual process interrupt, insert the subtitle, the subtitles have begun to force the adjustment or control of the audience to accept the angle and rhythm; and even directly to the content of the program and the communicator's understanding of the subtitles directly to the audience, this that is, directly guide the audience to decode; Moreover, the subtitles become the main body of the communication, the sound and the screen is only the footnote of the subtitles, like the network language symbol gradually derived from the expression package, expression package with the text is the main body of the spread.

Flower word seems to have entered the final stage of the development of subtitles, that is, the word is almost the main body of the spread, so some people on the network jokingly called "flower to save the reality show." From the perspective of information dissemination, the use of flower words is precisely the TV program from the control of communication information to meet the 
audience, once again back to the process of controlling the audience. But the word is to guide the audience to accept the way to complete the transmission of the perspective of the conversion, belonging to the acceptance of the type of transmission.

From Propagation to Acceptance: The Division of the Process School and the Symbolic School. In the early days of mass communication, television programs did not leave too much choice for the audience, and the majority of the programs to be disseminated could be accepted by the audience, which we could call the communicator-centered approach. The process school is concerned with the flow of channels and channels of information transmission, if the information in the dissemination of the process of loss or error in the receiving end decoding, it is considered a failure to spread. Thus the process school focuses on the source of the communication (communicator) and the media. The symbolic school argues that there is no misunderstanding of the decoding at the receiving end. If the communicator's message is not accurately transmitted to the receiver, it is because the communicator and the receiver are in a different text to understand the background. In other words, the information receiver and the communicator in an equal position, there is a symbol of re-interpretation and processing of the initiative.

Semiotics, there is no obvious active and passive relationship between communication and audience instead of coding and interpretation, interpretation is not a negative acceptance, but a positive behavior, because the formation of symbolic meaning can learn to develop The There are many forms of media mass in modern mass communication. People are in a mass knowledge network. The effect of television programs cannot be analyzed simply from the communicator and the transmission route, but also from the receiving end. Flower word to a certain extent, fully in line with the TV as a guide to the type of transmission. TV as a one-way spread of the form cannot do interact with the audience, now popular barrage through the subtitle form to make up for this point. However, the barrage is only a number of receiving end in the same media at the same time to express different forms of decoding information and cannot do the effective dissemination of information. It seems to return to the communicator as the center of the times, the difference is that the flower is based on the acceptance of the spread of the audience is to guide the transmission of the way.

From Coding to Decoding: The Recognition of Semiotics. The semiotics argues that the ambiguity and loss of information from the communicator to the receiver are caused by the different cognitive backgrounds at both ends, and that the encoding and decoding are not the same set of rules. TV programs will be sound, pictures and text and other large amounts of information into one, in the linear transmission path, each point there are multiple sources of information, the receiver decoding there is an unlimited number of possibilities. For example, Zhao Benshan's sketches, some people feel funny and some people feel that some people have discrimination. In the face of the same program, the results of decoding may be completely different. As others see the ban on the banana peel to feel funny, but if they stepped on a little but not funny.

The recipient will have different focus, understanding and acceptance depending on the background of the cognitive background. Information dissemination to be effective, you need to adjust the background according to the recipient's cognitive, this adjustment is not purely, but to guide. The emergence of fancy subtitles basically has the above form. The activation information of the flower word is to establish the cognitive background shared by the communicator and the receiver. Marking the focus information is the information that guides the receiver to select the attention, and the text and form of the word is the range of the selective understanding of the receiver the if the communicator and the receiver are in the same cognitive context, the communicator can "control" the recipient's selective focus and selective understanding of the way, 
then the "control" recipients to accept what and how to accept the logical. The "control" of the fancy subtitles is not propagated by the communicator as the center, but in the acceptance-oriented communication, which is also the idea of the symbolic school in the face of the information transmission process to produce information decay or disagreement.

\section{Acknowledgements}

Fund Project: Ministry of Education Humanities and Social Sciences Fund Project "The soft word grade standard for the Chinese international education" (16YJC740002)

\section{References}

[1] Zhang Jing: "TV Subtitle Classification", "Modern Communication: Journal of Communication University of China", No. 9, 2014, pp. 155-156.

[2] Fang Mei: "Syntactic means of Chinese contrasting focus", "Chinese language", No. 4, 1995, pp. 279-288.

[3] "From" the truth of the object "to" the truth of the sign ": On the semiotics of news seeking truth," International Journal ", No. 6, 2013, pp. 15-23.

[4] Zhang Zifan: "Choice: Screen or Text - Several Issues on TV Subtitles", "Modern Communication: Journal of Communication University of China", No. 2, 1998, pp. 90-92.

[5] Guo Zhenzhi: "Communication audience acceptance analysis", "Modern communication: Journal of China Communication University", 1994 No. 3, pp. 42-46. 